\title{
Adaptive Bandwidth Allocation in Wireless Networks with Multiple Degradable Quality of Service
}

\author{
A. Leelavathi ${ }^{1}$, G. V. Sridhar ${ }^{2}$ \\ Student M.Tech $(E C E)^{1}$, Associate professor ${ }^{2}$, ECE Department, AL-Ameer College of Engineering and \\ Information Technology, Gudilova, Anandapuram Visakhapatnam.
}

\begin{abstract}
In Wireless/Mobile networks various kinds of encoding schemes were used for transmission of data over a bandwidth. The desired quality and generated traffic varies with the requirement with this bandwidth. A generic video telephony may require more than $40 \mathrm{kbps}$ whereas a low motion video telephony may require about 25 kbps for data transmission. From the designing point of view these requirements demands for an alternative resource planning, especially for bandwidth allocation in wireless networks. In wireless network where bandwidth is a scare resource, the system may need to block incoming user if all of the bandwidth has been used to provide highest quality of service to existing users. However this bandwidth resource planning may be unacceptable for larger application. A degradable approach to multiple users can be made on bandwidth allocation to reduce the blocking probability without degrading the quality of service to existing users.

This work aims towards a realization of a wireless/mobile network using W-CDMA multi access technique supporting multilevel quality of services. The bandwidth allocation to multiple users is adjusted dynamically according to the required network condition so as to increase bandwidth utilization. The work analyze the performance deriving the degradation period ratio, mean degradation time and degradation state for the implemented wireless network.The proposed work is aim to implement on Matlab tool for its functional verification considering various mobility patterns
\end{abstract}

\section{Introduction}

Cellular wireless technology today has become the prevalent technology for wireless networking. Not only mobile phones but also other types of devices such as laptops and Personal Digital Assistant (PDA) can connect to Internet via cellular infrastructure. These mobile devices are often capable of running multimedia applications (e.g., video, images). Therefore, cellular networks need to provide quality of service (QoS) guarantee to different types of data traffic in a mobile environment. A call admission control (CAC) scheme aims at maintaining the delivered QoS to the different calls (or users) at the target level by limiting the number of ongoing calls in the system. One major challenge in designing a CAC arises due to the fact that the cellular network has to service two major types of calls: new calls and handoff calls. The QoS performances related to these two types of calls are generally measured by new call blocking probability and handoff call dropping probability. In general, users are more sensitive to dropping of an ongoing and handed over call than blocking a new call. Therefore, a CAC scheme needs to prioritize handoff calls over new calls by minimizing handoffdropping probability.

Again, bandwidth adaptation and scheduling are necessary mechanisms for achieving high utilization of the wireless resources (e.g., channel bandwidth) while satisfying the QoS requirements for

the users. These two techniques are closely related to call admission control, and in fact these three mechanisms jointly determine the call-level and the packet-level QoS for the different types of traffic in the cellular wireless air interface. For example, upon arrival of a new call or handoff call, bandwidth adaptation can be performed to degrade the channel allocations for some calls (still maintaining the QoS requirements) so that the new call can be admitted. Scheduling mechanisms impact the packet-level system dynamics (e.g., queuing behavior), and therefore, packet-level QoS. The packet-level dynamics can be exploited for designing efficient call admission control methods. The call admission control (CAC) and the adaptive channel adaptation (ACA) mechanisms are generally treated as the network layer (above layer-2) functionalities in the wireless transmission protocol stack. The scheduling and the adaptive modulation and coding (AMC) are layer-2 and layer-1 (i.e., physical functionalities, respectively.

\section{Qos In Wireless Communication}

Many real-time applications can use different encoding schemes according to their desired quality and generate traffic with different bandwidth requirements. For example, generic video telephony may require more than 40 KBPS, but low-motion video telephony requiring about 25 KBPS may be acceptable. From the standpoint of a system administrator, this property provides an alternative for resource planning, especially for bandwidth allocation in wireless networks. In wireless networks where the bandwidth is a scarce resource, the 
system may need to block incoming users if all of the bandwidth has been used up to provide the highest QoS to existing users. However, if these users can be degraded to a lower QoS level, it is possible to reduce the blocking probability without degrading the QoS of existing users to an "unacceptable" level. Various approaches and algorithms adopting this idea have been proposed. A graceful degradation mechanism is proposed in to increase bandwidth utilization by adaptively adjusting bandwidth allocation according to userspecified loss profiles. Thus, a system could free some bandwidth for new users by lowering the QoS levels of existing users.

\section{Problem Statement:}

The desired quality and generated traffic varies with the requirement with this bandwidth. A generic video telephony may require more than $40 \mathrm{kbps}$ whereas a low motion video telephony may require about $25 \mathrm{kbps}$ for data transmission these requirements demands for an especially Alternative resource planning for bandwidth allocation in wireless networks wireless network there bandwidth is scare resource, the system may need to block incoming user if all of the bandwidth has been used to provide highest quality of service to existing users. However this bandwidth resource this bandwidth resource planning may be unacceptable for larger application.

If these users can be degraded to a lower QoS level, it is possible to reduce the blocking probability without degrading the QoS of existing users to an "Unacceptable." Various approaches and algorithms adopting this idea have been proposed a graceful degradation mechanism is proposed to increase bandwidth allocation by adjusting bandwidth utilization by adaptively adjusting bandwidth allocation according user specified profiles. Thus, a system could free some bandwidth for new users. Sen et al proposed an optimal degradation strategy by maximizing a revenue function and Sherif et al [3] an adaptive resource algorithm to maximize band width utilization and attempted to achieve fairness with a generic algorithm. In terms of bandwidth utilization or service provider's revenue can be improved significantly by allowing QoS degradation.

\section{Approach}

In this work an optimal degradation strategy by adaptive resource allocation algorithm to maximize bandwidth utilization and attempted to achieve fairness in multi user communication. the system performance, in terms of bandwidth utilization or service provider's, can be improved significantly by allowing QoS degradation. Moreover, the potential dropping due to such cell crossings (i.e., handoffs) has to be taken into account. The forced-termination (or dropping) probability is a widely used metric to represent the compromise of QoS due to user mobility. This probability should be made as small as possible because admitting a user and then terminating his session before its completion would make the user even unhappier. In order to reduce this probability, many admission control algorithms give handoff users priority over new users.

In this work, the adaptive bandwidth allocation for QoS provisioning in wireless/mobile networks is presented. For a code division multiple access (CDMA) system, the wideband CDMA can be used for service degrade/upgrade; for a time division multiple access (TDMA) system (e.g., Bluetooth), service degrade/ upgrade can be achieved by an adequate assignment of time slots (i.e., polling policy)

\section{THE CONCEPT OF SPREAD SPECTRUM SYSTEM}

The capacity of any communications channel is defined by CE Shannon's channel capacity formula .......

$$
C=B w \log _{2}\left[1+\frac{S}{N}\right]
$$

\section{QOS MEASURES}

Degradation ratio (DR).The fraction of time a user receives degraded qos. Since we re considering multi

level Qos system,DR is defined as

$$
\mathrm{DR}=\frac{\sum_{i} \frac{\left(W_{m a x}-W_{i}\right)}{W_{m a}}, T_{i}}{\sum_{i} T_{i}},
$$

$$
\text { if a user receives level-i QoS for Ti seconds. }
$$

Throughput. It gives the account of number of packets arrived at received. It measures the efficiency of system.

Throughput= (number of bits received / number of bits send)*100

Degraded bandwidth (DB). It is measure of amount of bandwidth degraded from existing users. If number of users enters in the channel are increased then bandwidth degraded is increase. The degradation is stops for a user if he reaches the minimum bandwidth (b1). 


\section{MULTI PATH COMBINING:}

Multipath: reflection, diffraction, and dispersion of the signal energy caused by natural obstacles such as buildings or hills, or multiple copies of signals sent intentionally (soft handover)

Rake receiver used to combine different path components: each path is despread separately by "fingers" of the Rake receiver and then combined

Possible due to "low autocorrelation" of spreading code

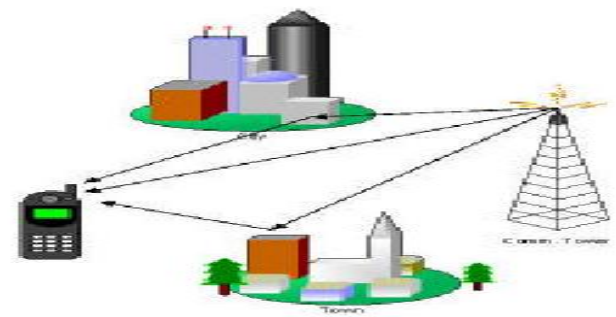

Fig: Multipath combining

\section{WCDMA TRANSMISSION MODULE:}

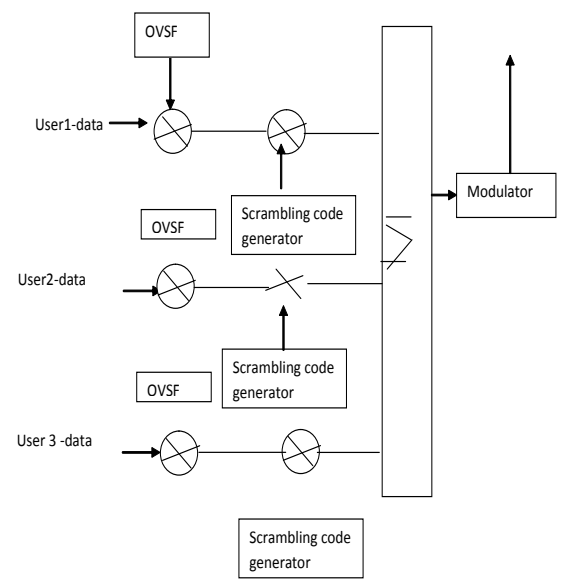

Fig: WCDMA Transmission Module

OVSF GENERATOR:

Transmissions froma single source are separated by channelization codes



\section{SCRAMBLING CODES:}

Scrambling codes make the direct sequence CDMA (DS-CDMA)technique more effective in a multipath environment

\section{LFSR (LINEAR FEED BACK SHIFT REGISTER):}

LFSR sequence through $\left(2^{\mathrm{n}}-1\right)$ states, where $\mathrm{n}$ is the number of registers in the LFSR. The contents of the registers are shifted right by one position at each clock cycle.

SUMMER: Adaptive power control schemes are employed in CDMA technology for efficient transmission of messages. 


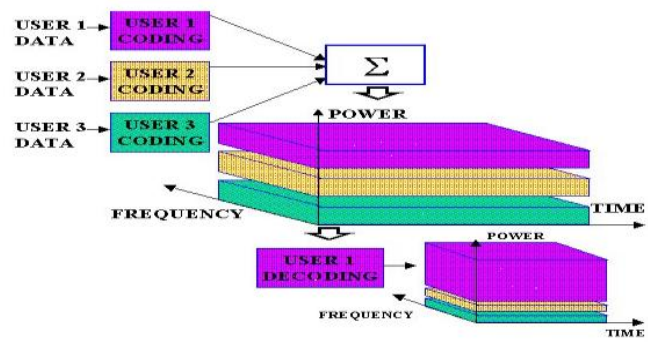

Fig:Summer

MODULATION:

The differentially coherent PSK (DPSK) signaling scheme makes use of a clever technique designed to get around the need for a coherent reference signal at the receiver.

\section{Result Analysis}
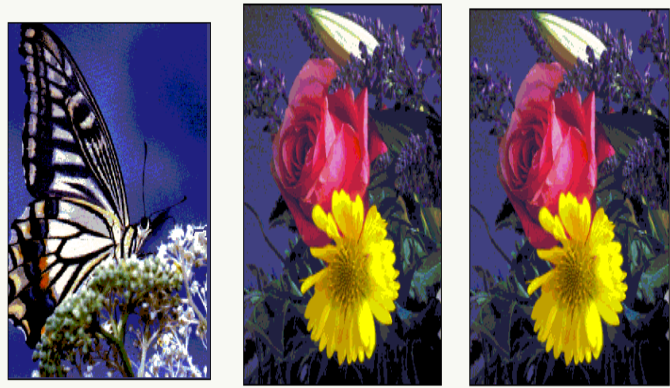

Fig 1 Three images

Fig 1 shows the original image considered for the three users in a group. Three users selects the image information which have then proposed using the communication system for transmitting the three images chosen are colored(256 level) bit map images.

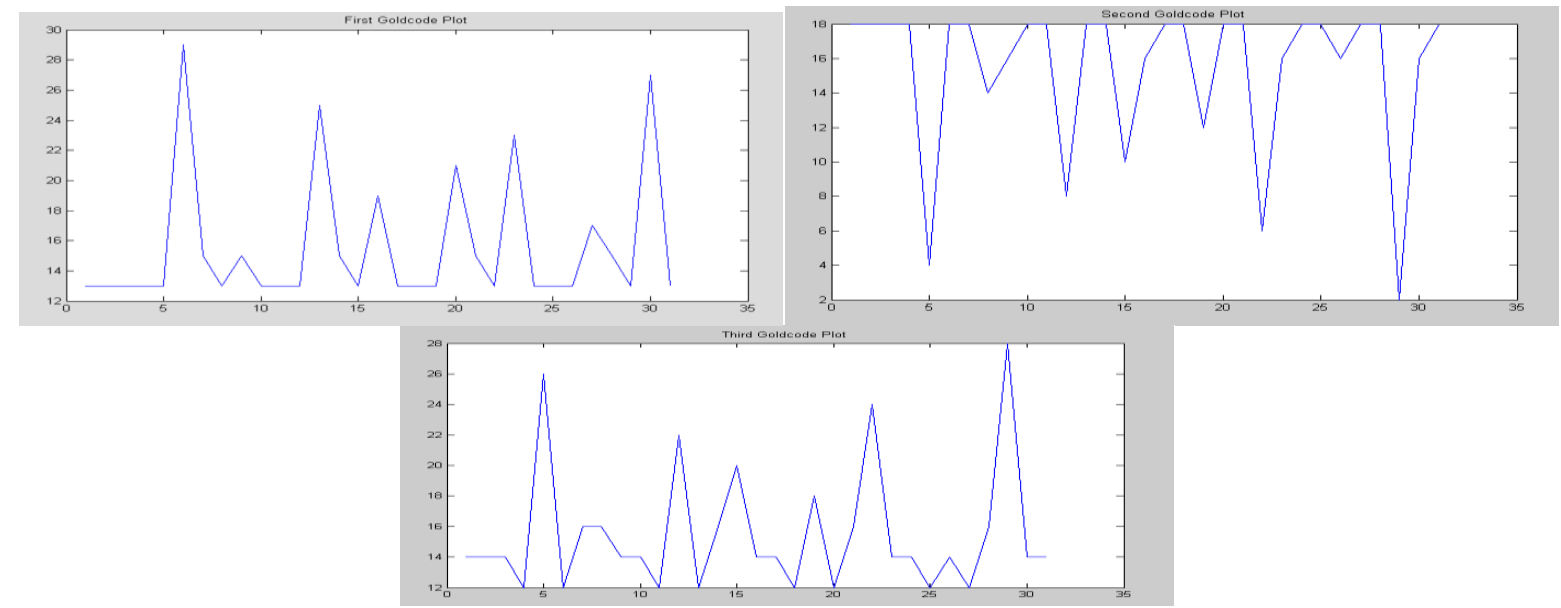

Fig 2,3,4 First, Second ,Third gold plot

Fig 2 shows the gold code generator due to correlation value obtained from comparison of two pn sequences with one varying in time shift version.

Fig 3The code generated is used as a spread code for user 2

Fig4 The code generated is used as a spread code and is used for user3 


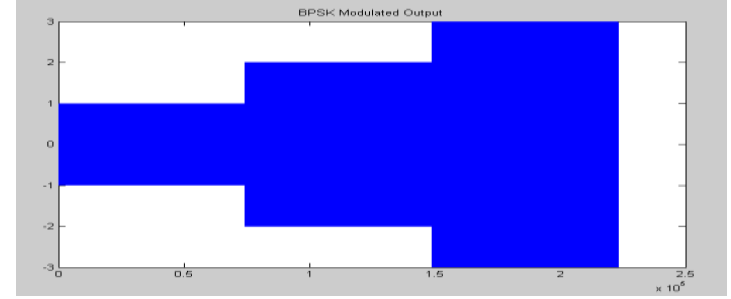

Fig 5 Bpsk modulated output

The Bpsk modulated data bundled with various power levels used before transmission. The three user data are modulated with sinusoidal carrier of o to $(2 *$ pi) sampled at 100 points.

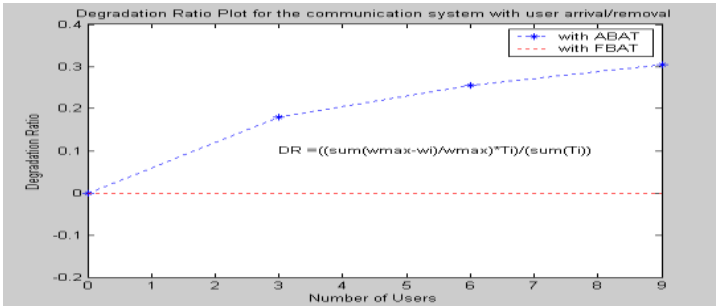

Figure 6 Degradation ratios for the implemented communication system shows the degradation the proposed two methods abat, fbat

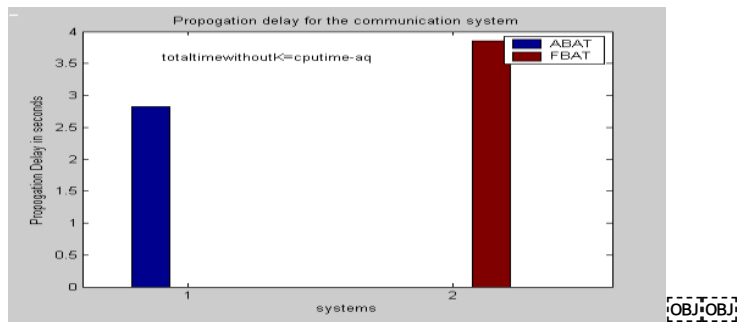

Figure 7 Propagation Delay comparison plot for the two implemented system shows the propagation delay observed for the two systems fbat and abat systems

\section{Conclusion}

In this paper, we derived an analytical model for a wireless network which uses adaptive bandwidth allocation to provide users multilevel QoS. Four performance metrics-Throughput, transmission time delay, degraded bandwidth, degradation ratio are observed.. The performance plots obtained gives that with increase in load with respect to time the through put level falls down because of increase in compression level which could be controlled by adaptive band width allocation method.

The performance is evaluated over WCDMA architecture by adding or removing different group of users to evaluate the algorithm efficiency. The metrics used to evaluate the performance are throughput, propagation delay, degradation ratio allocated bandwidth degradation is observed to outperform the existing fixed bandwidth allocation technique with more number of users it is seen that the delay for proposed abat method get reduced by $40 \%$ compared to fbat method.

\section{References}

[1] S. Sen, J. Jawanda, K. Basu, and S. Das, "Quality-of Service Degradation Strategies in Multimedia Wireless Network," Proc.IEEE Vehicular Technology Conf., vol. 3, pp. 1884-1888, May 1998

[2] S. Singh, "Quality of Service Guarantees in Mobile Computing," Computer Comm., no. 19, pp. 359-371, 1996.

[3] M.R. Sherif, I.W. Habib, M.N. Nagshineh, and P.K. Kermani,“'Adaptive Allocation of Resources and Call Admission Control for Wireless ATM Using Generic Algorithm,” IEEE J. Selected Areas in Comm., vol. 18, no. 2, pp. 268-282, Feb. 2000.

[4] T. Kwon, Y. Choi, C. Bisdikian, and M. Naghshineh, "Call Admission Control for Adaptive Multimedia in Wireless/Mobile Network," Proc. First ACM Int'l Workshop Wireless Mobile Multimedia, pp. 111-116, Oct. 1998

[5] S. Choi and K.G. Shin, "Location/Mobility-Dependent Bandwidth Adaptation in QoS-Sensitive Cellular Networks," Proc. IEEE Vehicular Technology Conf., vol. 3, pp. 1593-1597, 2001

[6] Y.B Lin, S. Mohan, and A. Noerpel, "Queuing Priority Channel assignment Strategy for PCS Handoff and Initial Access," IEEE Trans. Vehicular Technology, vol. 43, no. 3, pp. 704-712, Aug. 1994

[7] R. Ramjee, R. Nagarajan, and D. Towsley, "On Optimal Call Admission Control in Cellular Networks," Proc. IEEE INFOCOM '96, vol. 1, pp. 43-50, 1996.

[8] K. Mitchell and K. Sohraby, "An Analysis of the Effects of mobility on Bandwidth Allocation Strategies in Multi-Class Cellular Wireless Networks,” Proc. IEEE INFOCOM '01, vol. 2,pp. 1075-1084, 2001.

[9] A. Sutoving and J.M. Peha, "Novel Heuristic for Call Admission Control in Cellular Systems," Proc. IEEE Int'l Conf. Universal Personal Comm., vol. 1, pp. 129-133, 1997 